\title{
Is Sustainability a Pattern of Solution in a Functional State Space?
}

\author{
Andy E. Williams, Nobeah Foundation, Nairobi, Kenya
}

\begin{abstract}
:
Human-Centric Functional Modeling has been proposed as a universal approach towards defining representations of the behavior of systems. Such descriptions are hypothesized to be complete in terms of defining all behavior the system is capable of displaying. Human-Centric Functional Modeling has been used to model the behavior of the cognitive system in terms of a functional state space, and how problems of sustainability or problems related to sustainable development might be represented in this functional state space of the cognitive system. This paper explores solutions in this functional state space and the questions that must be asked in considering whether and how such solutions might radically increase capacity for sustainability.
\end{abstract}

\section{Introduction}

There is an established tradition in using "state spaces" for semantic modeling, such as semantically modeling scientific or other theories [1], or in this case semantically modeling sustainability patterns. While the philosopher Bas C. van Fraassen has been attributed credit [2] for the concept of the "state space" as a semantic modeling approach, a number of researchers citing his work have taken the concept of "state spaces" similar to those described in this paper and applied them to all of science in general [3]. The difference appears to be that this paper has made the additional refinement of using the human system, particularly the cognitive system, as a basis for this state space modeling approach, so that these models could be understood intuitively without the need to understand any logical frameworks at all. Hence the name "Human-Centric Functional Modeling" or HCFM. Use of this functional state space is predicted to enable us to exponentially increase our ability to understand and navigate complexity in any system it is used to represent, and is predicted to exponentially increase our ability to solve problems in these systems where not otherwise reliably achievable [4]. It then follows that using functional state space to model information is predicted to significantly increase our capacity to understand and validate theories in sustainability.

Human-Centric Functional Modeling or HCFM [5],[7] represents the cognitive system as a space of concepts connected by reasoning processes that act as functions which enable the cognitive system to transition from one concept to another. This representation of cognition as moving through a "conceptual space", and this representation of the collective cognition as moving through a "collective conceptual space" has been used to define a model of collecitve cognition (General Collective Intelligence or GCI [6]). This conceptual space is an example of a "functional state space", which is used in Human-Centric Functional Modeling to describes systems as having a set of functions through which the system might transition from one state to another, where each state is defined in terms of which functions are available to transition to other states. These states described in terms of functions are referred to as "functional states", each of which might be composed of other functional states. All of the functions of a system within a given domain of behavior map between functional states belonging to the same category. Any given category of functional states then forms a "space" of functional states or a functional state space.

\section{Problems in Sustainability as Gaps in the Collective Conceptual Space}

There are a number of problems related to sustainable development that groups can't reliably solve. A list of some of these problems is provided in table 1.

\section{Problem Name Description and Example}


Alignment of Choices The problem of misaligned incentives. An example is "The Problem of with Individual Interests Famine". In many cases we not only tend to solve the wrong problem, but also tend to be constrained to do so. To understand why, imagine there is a famine that has left you and your family starving in South Sudan. Somewhere in Brussels, a program manager at a large humanitarian organization is deciding on what aid to send in response. Before deciding to send that aid, the program manager might go over the organization's long list of policies and procedures, perhaps making sure that any food aid meets the recommended minimum daily allowances of nutrients, perhaps making sure it comes from an approved supplier, perhaps making sure it comes from factories that meet its labor standards, and so forth, before finally sending three days worth of food aid after that process is concluded. Rather than considering all of this, you in South Sudan on the other hand might have preferred to just take the money equivalent to that three days worth of aid. Anything big donor organizations do is expensive. So with that money you might have been able to simply buy a month's worth of cornmeal flour to make ugali (a type of cornmeal porridge) from a passing trader that same day. Ugali is largely starch, and might not meet the donor organization's daily nutrient requirements. But it is familiar to the primarily starch based diet in rural Africa where meat is harder to come by, and will protect your family from starvation. Or you might have preferred to take the money for that three days worth of aid and simply travel with your family to stay with relatives in neighboring Kenya, where instead of eating for three days, you and your family might be fed and sheltered for the next six months. In this way both of these options might result in levels of food security that are an order of magnitude or more greater. But these options can't be selected regardless of how much better they are. Because each of you are solving a different problem. You are solving the problem of the famine. The program manager is solving the policies of his organization. Solving this problem of misaligned interests requires decision-making by decentralized agents that can't become aligned with any centralized interests.

Better Choices are Not The problem of the inability to compare options and reliably select best one. An Useful example is "The Problem of Finding the Better Mousetrap". The author's first project with the Nobeah Foundation was to develop an early prototype of a radically cost effective hybrid educational laptop/e-reader called the Afripad in response to the $\$ 300$ M USD tender offered by the government of Kenya. At about the cost of textbooks for one year the Afripad had the potential to address the lack of learning materials in African schools when combined with free or low-cost e-textbooks. The author was able to attract somewhere around 20,000 people to apply to work with the Nobeah Foundation as volunteers to implement this and other projects. Of these applicants we brought on a staff of around 200 mainly volunteer staff. The Nobeah Foundation had partnerships with the leading vendors in that space, including a partnership with Datawind, the world's leading manufacturer of low cost computers. The project had the endorsement of the two top engineering universities in the country, as well as the endorsement of the governors of multiple counties in Kenya. Through engaging 40-50 engineering interns from those universities to help design the computer, as well as to help design the solar powered charging stations and other infrastructure, some of which were patented, the project demonstrated the capacity to build the computer locally, rather than to build it in Shenzen or some 
Shortcomings of Systems Science Methodologies are Invisible

Lack of Coherence in Group Reasoning

The Problem of Direction other city in China and merely assemble it locally as the other competing projects planned to do. Because of this, the project was estimated to create tens of thousands more jobs than the competing bids. Since the computer was also targeted at a cost that was a tenth of that of the computers being offered by the other bids, it was thought that the Afripad had such a better value proposition that it could not lose the award. However the Afripad was not even selected for the second round. Up until now, many years later, the winning bidders have not successfully delivered the project and the project has been widely criticized as corrupt and failed. Through all these developments it became clear that the solution to radically increasing social impact in Africa was not "building a better mousetrap". Instead the solution was building a system of decisionmaking capable of reliably selecting and funding the best mousetrap. But no decision system can improve things if it isn't used. Solving this problem requires a system of organization that out-competes other systems so that it is reliably used.

There are a number of patterns of problems in applying systems thinking that anecdotal observation suggests are very prevalent. These patterns might severely hamper the ability of systems thinkers to make useful contributions to understanding and navigating the complexity of systems, as opposed to merely succeeding in selling courses in systems thinking programs at their universities. An example of these patterns is not understanding the problems that cannot be solved with a given systems thinking methodology. For example, presented with a new idea that must be evaluated, an individual might respond saying essentially "I don't believe that because I've never heard that before", or without reading the justification for a given set of claims they might state "those claims are far-fetched", all of which equate to "I will consider the idea and claims when the consensus of opinions among others agrees with them". This is an example of type 1 (fast or intuitive) reasoning which determines truth or falsehood using intuition, which relies on patterns of solutions observed in the past, one of which is group consensus. However, type 1 reasoning is incapable of solving problems requiring that new methods of calculation be used. Individuals have both type 1 (fast or intuitive) reasoning and type 2 (rational methodical or slow) reasoning. Type 1 reasoning is necessary for solving uncomputable problems, and type 2 reasoning is necessary in solving computable ones. Type 1 and type 2 reasoning come to different conclusions, but individuals can switch between type 1 and type 2 reasoning depending upon which is optimal. Therefore individuals can reliably converge on a conclusion. However, some individuals are prone to type 1 reasoning in certain circumstances in which other individuals are prone to type 2 reasoning. This leads to polarization in groups. Without a "collective cognition" that is able to switch the group from type 1 reasoning to type 2 reasoning or vice versa, groups cannot reliably converge on optimal collective reasoning. In this sense groups without collective cognition lack coherence in group reasoning. The problem of the inability to discern which direction provides the most productive path forward in the case of wicked problems. Where an interaction between three components of an ecosystem is results in some function that solves a problem, that problem might be reliably definable, and that solution might be reliably discoverable. For example, a problem defined by by the interaction between the roots of a tree, the soil, and bacteria, might be reliably 
The Problem of Complexity in Chains of Cooperation definable, and the solution required for the tree to bear healthy fruit might be reliably discoverable by agricultural researchers. On the other hand, where the interaction is between one thousand components of the ecosystem, that problem might not be reliably definable and the function providing the solution might not be reliably discoverable. The chain of cooperation between components that is required to define or to solve the problem represents a path in "functional state space" that is too long and therefore too complex. Combining sustainable development projects into value chains that cooperate might exponentially increase outcomes. As described in the "South Sudan School Uniforms Value Chain" example, a value chain of only 5 projects might increase outcomes $750 \mathrm{X}$ per program dollar. But in order to achieve this impact the combination of projects into chains of cooperation that can significantly increase impact must be a clear direction forward. In order for there to be a clear direction forward there must be a more general way of assessing impact so that the impact of different directions forward can be compared.

The problem that complex cooperation is required to radically increase ability to achieve collective outcomes, but cooperation becomes more unlikely the more complex it becomes. An example is "The Problem of Value Chains". Combining sustainable development projects into value chains that cooperate can exponentially increase outcomes. As described in the "South Sudan School Uniforms Value Chain" example, a value chain of only 5 projects might increase outcomes $750 \mathrm{X}$ per program dollar. But the more complex the value chain, the less likelihood that prospective participants will understand it and actually participate. If a value chain of 5 projects is very difficult to launch, a value chain of 10,000 projects is impossible. Finding chains of cooperation that exponentially increase outcomes is only part of the problem. The problem is also decoupling the chains so participants only need to understand their link in the chain in order to decide whether to participate. This problem of inability to enter into chains of cooperation that are too complex has implications regarding the length of reasoning, and therefore the complexity, that models of AGI or GCI (General Collective Intelligence) can navigate, wherever those models are based on decentralized components that cooperate to achieve an outcome. This also determines which problems cannot reliably be solved (i.e. it determines which problems are "wicked problems"). 
Table 1: List of the categories of problems that can't reliably be solved in sustainability and sustainable development.

If solutions are defined in conceptual space as reasoning which navigates a path from one concept to another, these are gaps in the collective conceptual space in that collective reasoning can't reliably navigate to these regions.

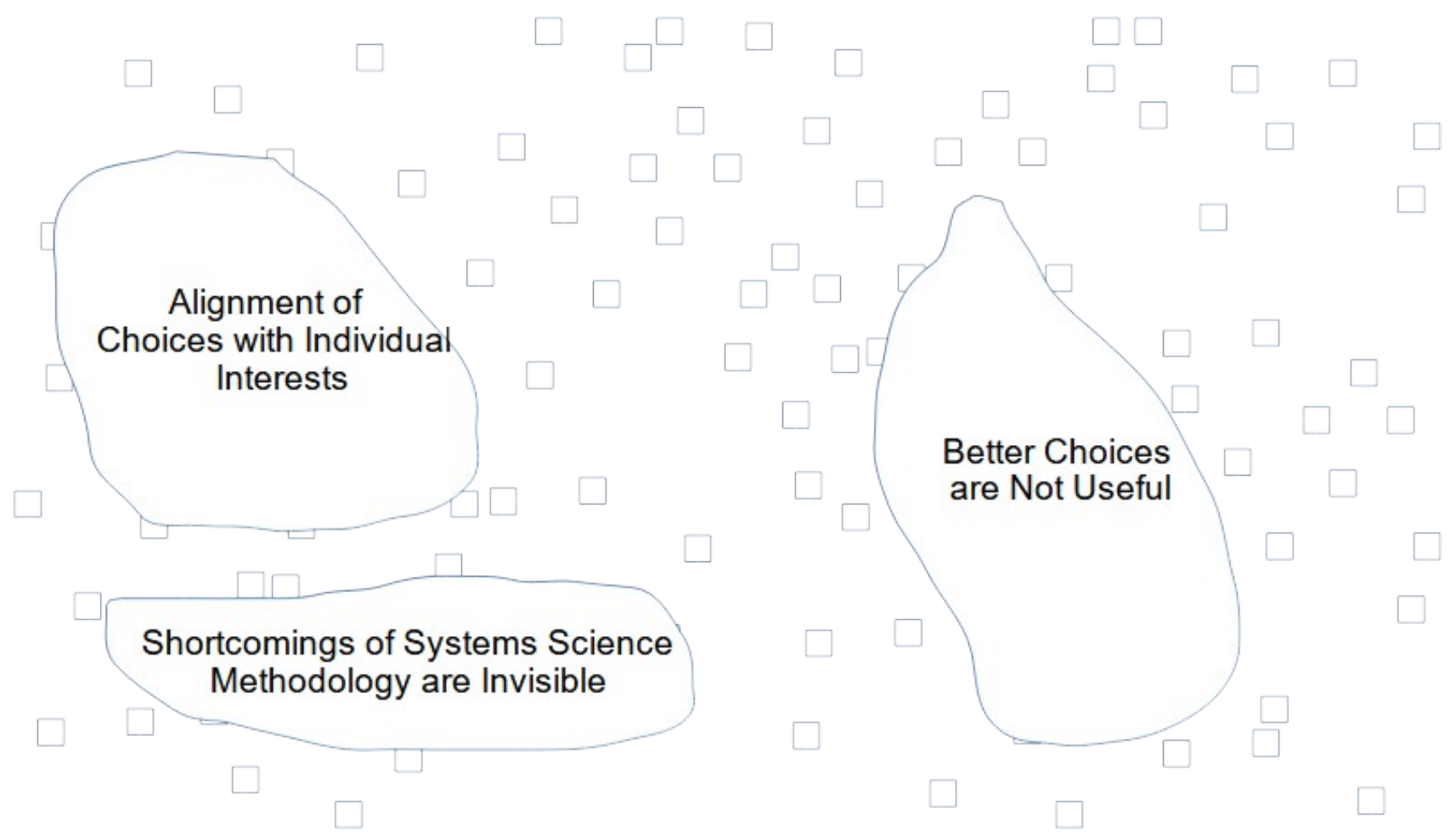

Figure 1: Gaps in the collective conceptual space which collective reasoning can't reliably navigate, representing problems that groups can't reliably solve and solutions that groups can't reliably discover.

Validating the Hypothesis of Sustainability Being a Pattern in Functional State Space

If sustainability is a pattern in some functional state space how is that space defined and what does that pattern look like? Is sustainability a pattern in the collective conceptual space that serves as the functional state space of the collective cognition? Is sustainability a pattern in some functional state space that defines the economic state of each individual in the group? Though it is still very powerful in its current form, the idea of conceptual space is incomplete. There are a number of problems that must be solved before any implementation of a conceptual space can be used to semantically store concepts and reasoning that might be searched to find solutions to sustainability problems. One is that distances are not precisely quantified. Another is that there is not yet a representation of concepts and reasoning relationships that is fully self-contained (independent of any lookup table). In the mean time we must use the subset of conceptual space that can be represented with ontologies, and we must accept the limitations of working with ontologies as opposed to working with a complete semantic representation. However, because any functional state space might be represented by a graph containing a network of nodes representing functional states connected by edges representing the processes through which the system transitions between states, then solving these problems for any functional state space solves these problems for every functional state space, including the conceptual space. 


\section{Navigating the Gaps in Conceptual Space Related to Sustainability}

In this GCI platform, collective reasoning problems concerning how to achieve collective outcomes targeted by the collective reasoning of a group (problems requiring collective reasoning processes to be directed at achieving specific collective outcomes) are approximated as one of three types: closed problems solvable with chains of collective reasoning involving existing concepts, closed problems solvable with chains of collective reasoning involving new concepts, and open problems solvable with new generalizations of concepts or more detailed specialization of concepts that are incorporated into new concepts used in chains of collective reasoning. Classifying these reasoning problems into these patterns enables all solutions to be readily compared in terms of their projected impact on the targeted outcome using the same general method of comparison for each pattern and outcome.

An example of the first pattern is the proposed "Social Impact Marketplace" platform in which the goal is to orchestrate cooperation to define value chains of businesses that cooperate to meet local demand while vastly increasing collective social impact through one business agreeing to buy a key product or service from one business in the value chain in return for another business in the value chain agreeing to buy from them. In this example the product or service exchanged between one business and another represents the "concept", and the set of interacting businesses represent the chain of cooperation that is conceptualized by a chain of collective reasoning. Classifying these reasoning problems into these patterns enables all solutions to be readily compared in terms of their projected impact on the targeted outcome using the same general method of comparison for each pattern and outcome. enables all individuals in a group to collaborate in suggesting such solutions, and enables all individuals to collaborate in evaluating which solution is predicted to be the best. Because solutions can be defined, objectively compared, and selected by exponentially more people, rather than being defined, subjectively compared, and selected by only a few people, then the ability to search an exponentially greater number of such solutions in order to select the best one can be demonstrated. It is hypothesized that this ability to search an exponentially greater number of solutions might equate to the ability to search an exponentially greater region in the collective conceptual space.

Each of the three patterns of solution is represented as a set of concepts that are described by an ontology. This ontology forms some subset of the conceptual space defined by Human-Centric Functional Modeling. The many instances of proposed solutions that are defined within that ontology form an even larger subset of the conceptual space, so that by defining more and more problems with these patterns, and by discovering more solutions with these patterns, a larger and larger subset of the conceptual space might be represented.

Human-Centric Functional Modeling represents collective reasoning as navigating the collective "conceptual space" in a way that leads to general problem-solving ability for the group. Each reasoning process has a projected fitness in achieving some targeted function, as well as a target fitness in achieving that function, and an actual fitness in achieving that function. This projected, targeted, and actual fitness defines a general "fitness space". General problem-solving ability is represented as the potential ability to navigate the entire collective conceptual space in a way that is dynamically stable in this "fitness space" that describes the fitness of the cognitive system to execute all of its reasoning functions ("cognitive fitness"). This dynamic stability is achieved through ensuring that in selecting and executing reasoning processes the cognitive system follows a set of equations that are stable in the three dimensions of fitness space. One set of equations which exhibit dynamical stability in three dimensions and which might be used to define such an intelligence algorithm are the Lorenz equations for convection [9]. 
In the "Social Impact Marketplace", which is proposed to be the first implementation of GCI, the conceptual space is approximated by three ontologies, with each ontology representing one of the three patterns of solution by which sustainability or other impact might be exponentially increased. For each pattern of solution the platform automates the collaboration required to select the value chain that maximizes targeted outcomes. In doing so the algorithm creates the ability to overcome the limitations in group problem-solving ability representing the gaps in the collective conceptual space that can't reliably be navigated today. To do so the navigates the conceptual space with an algorithm that is intended to approximate general problem-solving ability [9]. Take the case in which $\mathrm{N}$ potential participants in cooperation must select between $\mathrm{M}$ processes of cooperation, where $\mathrm{M}$ is greater than N. Or more specifically, the case in which $\mathrm{N}$ potential participants must select between $\mathrm{M}$ value chains of businesses. Centralizing the decision of which chain of cooperation (value chain) will be used so that it is made by any single individual might align the decision with that individual's interests, rather than with maximizing collective fitness. Such centralization would then represent individuals reasoning individually within a group, rather than representing collective reasoning. The collective reasoning process in this platform requires each individual to suggest a solution to achieving a targeted outcome, where that solution fits one of these three patterns. The platform then enables each individual to bid to participate in some chain of cooperation proposed by any individual on the platform. The individual with the first role in the chain of cooperation selects the top N1 chains of cooperation from their perspective, the second individual selects the top $\mathrm{N}-2$ chains of cooperation from their perspective, and so forth until the Nth individual selects the final chain of cooperation that all $\mathrm{N}$ actors participate to execute.

\section{Conclusions}

Understanding what a "functional state space" is, and it is important in solving wicked problems in sustainability begins with considering our minds as cognitive systems to be represented as moving through a space of thought. Consider this space of thought to be a functional state space since each point in it represents a concept and since each concept is a functional state in that it is defined by the reasoning processes that function to transition the mind from it to other concepts. If our cognitive systems can be represented as moving through a space of thought, then it is possible to represent all other systems as moving through their own subset of this thought space. This subset of thought space is a functional state space that the system we are thinking about moves through as our thoughts about it change. Alternatively, completely independently of us, any system can be represented as moving through its own functional state space as it changes, regardless of whether or not we think about it. All systems then move through such a functional state space, either in our minds or in the real world.

If everything we can conceive of in our thoughts falls within a functional state space, and if we can conceive of entire economic systems, then it is possible to represent civilizations economically as moving through their own functional state space as well. Assume that this functional state space allows us to create a mathematical definition for every property of sustainability where that definition can be expressed in terms of movement through this collective conceptual space as the functional state space of the collective cognition. Since every other system potentially also moves through a functional state space, and since these properties belong to functional state space rather than to any particular system, then the same expressions regarding sustainability might be used to understand sustainability in all other systems as well. Sustainability in the collective conceptual space is hypothesized to be represented by the ability to navigate the entire collective conceptual space so as to be able to access the currently unreachable regions where sustainable solutions are expected to reside. Sustainability in an economic or other system is hypothesized to be represented by the exact same expression in the 
functional state space of the system. The importance of having a universal way of understanding all systems is that it enables our understanding of any one system to potentially be reused in understanding any other. The importance of understanding cognition in this way is that it allows us to define the concept of a collective cognition called "General Collective Intelligence" [6], and that it allows us to understand why poverty, climate change, and other wicked problems in the world might not be reliably solvable without this collective cognition [7], but might be reliably solvable with it [8].

\section{References}

[1] Suppe, Frederick. The semantic conception of theories and scientific realism. University of Illinois Press, 1989.

[2] Lloyd, Elisabeth A. "A semantic approach to the structure of population genetics." Philosophy of Science 51.2 (1984): 242-264.

[3] Niiniluoto I. (1987) Quantities, State Spaces, and Laws. In: Truthlikeness. Synthese Library (Studies in Epistemology, Logic, Methodology, and Philosophy of Science), vol 185. Springer, Dordrecht. https://doi.org/10.1007/978-94-009-3739-0_3

[4] Williams, A. E. (2020, July 11). Human Intelligence and General Collective Intelligence as Phase Changes in Animal Intelligence. https://doi.org/10.31234/osf.io/dr8qn

[5] Andy E. Williams, A Revolution in Systems Thinking?, Proceedings 2021 Congress of the World Organization of Systems and Cybernetics (WOSC), in press (2022)

[6] Williams, A. E., Defining a Continuum from Individual, to Swarm, to Collective Intelligence, to General Collective Intelligence, International Journal of Collaborative Intelligence, in press (2022)

[7] Williams, A.E. (2021) Are wicked problems a lack of general collective intelligence?. AI \& Soc (2021). https://doi.org/10.1007/s00146-021-01297-8

[8] Williams, Andy E. Is General Collective Intelligence a Reliable Path Towards Achieving Green Growth, International Journal of Green Economics, in press (2022)

[9] Williams, A. E., Approximating an Artificial General Intelligence or a General Collective Intelligence, International Journal of Collaborative Intelligence, in press (2022) 